\title{
Electrically induced spin resonance fluorescence. I. Theory
}

\author{
A. Nogaret* \\ Department of Physics, University of Bath, Claverton Down, Bath BA2 7AY, United Kingdom \\ F. M. Peeters \\ Department of Physics, University of Antwerp, Groenenborgerlaan 171, B-2020 Antwerpen, Belgium
}

(Received 21 February 2007; revised manuscript received 25 May 2007; published 8 August 2007)

\begin{abstract}
We calculate the fluorescence of electron spins confined to a plane and driven into resonance by a magnetic field gradient and a constant magnetic field applied at right angles to each other. We solve the equation of motion of two-dimensional electrons in the magnetic field gradient to derive the dispersion curve of spin oscillators, the amplitude of electron oscillations, the effective magnetic field sensed by the electron spin, and the rate at which electrons are injected from an electrode into spin oscillators. We then switch on the interaction between the spin magnetic dipole and the electromagnetic field to find the fluorescence power radiated by the individual spin oscillators. The rate of radiative decay is first derived, followed by the probability of sequential photon emission whereby a series of spontaneous decays occurs at random times separated by intervals during which the spin performs Rabi oscillations. The quantum correlations between random radiative decays manifest as bursts of emission at regular intervals along the wire. We integrate all multiphoton processes to obtain an exact analytical expression for the radiated electromagnetic power. The present theory obtains all parameters of the problem including magnetodipole coupling, the particle dwell time in the magnetic field gradient, and the spin polarization of the incoming current. The output power contains a fine structure arising from the anharmonicity of electron oscillations and from nonlinear optical effects which both give satellite emission peaks at odd multiples of the fundamental frequency.
\end{abstract}

DOI: 10.1103/PhysRevB.76.075311

PACS number(s): 85.75. $-\mathrm{d}, 73.23 .-\mathrm{b}, 76.20 .+\mathrm{q}$

\section{INTRODUCTION}

The fluorescence of a quantum transition excited by a resonant electromagnetic beam ${ }^{1}$ has been an important means for investigating the atomic structure ever since the experiment of Lamb. ${ }^{2}$ Photons generated by spontaneous decay provide information on the population of energy levels, interactions between atoms via super-radiant emission, and the density of states of the vacuum. In particular, the fluorescence of electron spin resonance has been the subject of intense study ${ }^{3}$ whose results stimulated the development of the dressed atom approach in quantum field theory. ${ }^{4}$ Different regimes of fluorescence have been demonstrated by using electromagnetic cavities to change the rate of spontaneous decay relative to the oscillator strength of quantum transitions. ${ }^{5}$ Thanks to advances in semiconductor growth techniques, resonant light-matter interactions have been observed between optical cavity modes and the quantum transitions of a quantum well. ${ }^{6}$ By contrast, spin resonance fluorescence has proved difficult to observe in the conduction band of semiconductors ${ }^{7}$ because of the necessity for doping which broadens the emission line.

In the present paper, we describe a simple physical system where spin resonance is excited by the application of a direct voltage. As no prior irradiation is necessary, the system effectively converts direct electric power into continuous wave fluorescent emission. We develop a theory aimed at calculating the electromagnetic power radiated. We first describe the dynamics of electrons in inhomogeneous electric and magnetic fields within a semiclassical picture. We show that charge oscillations become interdependent of the spin motion. This results in a system characterized by a single degree of freedom which sets the frequency of electron oscillations or equivalently, the frequency of spin resonance. We refer to this system as a spin oscillator. We then calculate the fluorescence of spin resonance by computing the interaction of a two level quantum system with the field and by assuming coupled resonant oscillators which oscillate in phase. We compute the probability of photons being emitted in sequence which, under specific conditions, dramatically enhances the microwave output power. The theory is valid for all regimes of spontaneous emission and obtains all physical parameters of the system. Approximations of the theory for different regimes of emission are given and discussed.

The paper is organized as follows. Section II describes the dynamics of spin oscillators in a gradient of magnetic field. This yields both the oscillator dispersion curve and the rate at which electrons are injected from the electrode into individual oscillators. Section III reports the renormalization of the energy and lifetime of spin states caused by the interaction of the spin system with the field. We derive the rate of spontaneous decay and plot its time dependence for a spin oscillating in the magnetic field gradient. Section IV calculates the probability for the sequential emission of multiple photons and photon antibunching. We then sum all possible processes and derive an exact expression for the microwave power output by the magnetic field gradient region. Section $\mathrm{V}$ comments on the theoretical results and the assumptions on which they are based. Section VI is a summary of our findings.

\section{ELECTRON DYNAMICS IN A MAGNETIC FIELD GRADIENT}

We consider electrons confined to a two-dimensional plane and modulated by a spatially variable magnetic field. 


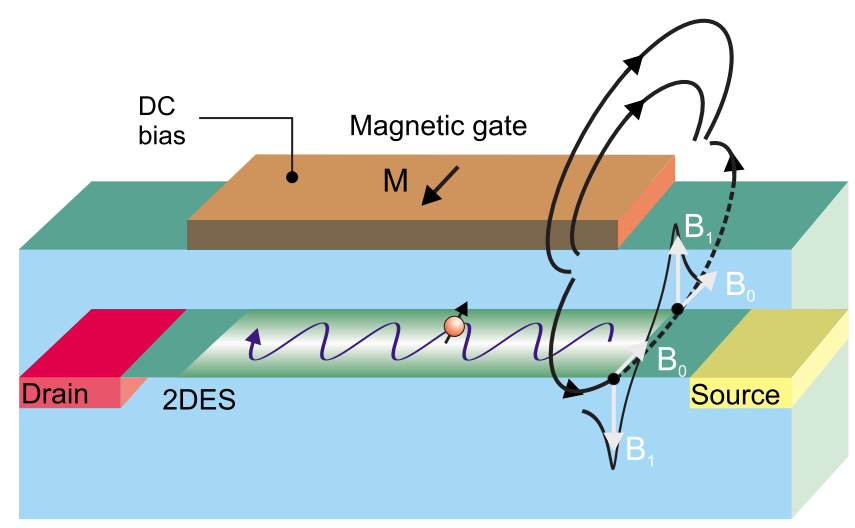

FIG. 1. (Color online) Electrically induced electron spin resonance in a semiconductor quantum well subjected to crossed magnetic fields. The fringe magnetic field emanating from the stripe has a vector component $B_{1}$ normal to the two-dimensional electron gas and a vector component $B_{0}$ in the plane. The $B_{0}$ component is essentially constant over the 2DEG, while the $B_{1}$ component changes sign at the center of the channel. A drain-source current is applied which causes electrons to oscillate in the gradient of magnetic field $B_{1}$ and to radiate microwaves. A bias applied to the magnetic gate modifies the oscillator frequency hence the emission frequency.

The fabrication of small magnetic elements at the surface of a quantum well ${ }^{8-11}$ provides a simple route for achieving such modulations. Figure 1 shows the section of two-dimensional carriers covered by a rectangular magnetic stripe magnetized perpendicular to the channel. Its stray magnetic field threads the two-dimensional electrons system with a vector component $B_{0}$ in the plane and a second magnetic field vector component $B_{1}$ perpendicular to the plane. We shall assume that these magnetic fields only affect the rectangular area of the two-dimensional electron gas (2DEG) underneath the magnet and are zero everywhere else. Within this area, which we refer to as the magnetic waveguide, $B_{0}$ is constant and $B_{1}$ varies linearly in the transverse direction ( $z$ axis). We write $B_{1}=b z$, where $b$ is the magnetic field gradient. The center of the channel at $z=0$ is the boundary where the two regions of opposite magnetic fields meet. Across this line, the magnetic potential has a double potential well which confines electrons in magnetic edge states. These allow a drainsource current to flow without dissipation. Reflections at the edges of the magnetic well cause a transverse oscillatory motion which combines with the longitudinal drift into snakelike orbits. ${ }^{12}$ In Fig. 1, the strength of the magnetic confinement is limited by the strength of the ferromagnet. We will calculate the amplitude of largest oscillations and show that it is comparable to the distance separating the magnetic poles. This means that magnetic edge states with smaller amplitude will be unaffected by the finite width of the 2DEG. This also makes the magnetic field gradient approximation appropriate as real structures only have a constant magnetic field gradient between their poles. ${ }^{13-16}$ Magnetoelectric edge states will also form at the edges of the channel; however, these experience magnetic fields keeping the same sign which give none of the spin resonant effects of interest here.
Magnetic edge state trajectories are bent by the Lorentz force and by the Zeeman force which pulls the electron magnetic dipole moment. The latter results in the weakest effects of the two forces but it nevertheless controls motion at the center of the channel where the oscillations are the fastest. The Zeeman force oscillates at the frequency of Larmor precession because it is proportional to the $\mu_{x}$ component of the magnetic dipole moment and this quantity oscillates at the Larmor frequency. Given that the precession motion is about $B_{0}$, the Larmor frequency is given by $\omega_{0}=-\gamma B_{0}$, where $\gamma$ is the spin gyromagnetic ratio. When the frequency of transverse oscillations and the Larmor frequency matches, the Lorentz force is magnified by the Zeeman force. Since the Lorentz force depends on the magnetic field whereas the Zeeman force depends on the magnetic field gradient, one finds a band at the center of the channel where oscillations are mainly driven by the Zeeman force. The point at which both forces balance is

$$
l_{\text {Zeeman }}=\left(\frac{g^{*} m^{*}}{g_{0} m_{0}}\right)\left(\frac{1}{k_{F}}\right),
$$

where $k_{F}=\sqrt{2 \pi n_{s}}$ is the Fermi wave vector in the 2DEG, $n_{s}$ is the electron density per unit area, $g^{*}$ and $m^{*}$ are the Landé factor and the electron effective mass in the $2 \mathrm{DEG}$, and $g_{0}$ and $m_{0}$ are the Lande factor and mass of the free electron in vacuum. In most semiconductors, $l_{\text {Zeeman }}$ is of the order of $0.4-4 \mathrm{~nm}$. Since the maximum amplitude of oscillations is on the scale of a few hundred nanometers, the pull on the spin will generally be negligible. As a result, it is sufficient to compute the dynamics of a Fermi electron by only considering the action of the Lorentz force. ${ }^{17} \mathrm{~A}$ semiclassical picture gives an appropriate level of description to the problem. ${ }^{18,19}$ Starting from Newton's equation of motion, we obtain by integration the vector components of the velocity at the Fermi level:

$$
\begin{gathered}
\tau_{b} \dot{Y}=\cos (\theta) / 2-Z^{2}, \\
\left(\tau_{b} \dot{Z}\right)^{2}=\left[\cos ^{2}(\theta / 2)-Z^{2}\right]\left[Z^{2}+\sin ^{2}(\theta / 2)\right],
\end{gathered}
$$

where we introduce the dimensionless coordinates $Y \equiv y / l_{b}$ and $Z \equiv z / l_{b}$ which are expressed in units of $l_{b}$ $\equiv 2 \sqrt{\hbar k_{F} /(e b)}$. Similarly, the characteristic time scale of the system is $\tau_{b} \equiv m^{*} / \sqrt{\hbar k_{F} e b}$. It is easy to show that $l_{b}$ is the maximum swing an oscillator can have and that $\omega_{c}=1 / \tau_{b}$ is the maximum angular frequency an oscillator can have. The quantity $\theta$ which parametrizes the different oscillators arises from the constant of integration which states that at $Z=0$ the electron velocity is $\left\langle 0, v_{F} \cos (\theta), v_{F} \sin (\theta)\right\rangle$. Hence, $\theta$ is the angle at which an orbit crosses the line $B_{1}=0$. Typically for an InAs quantum well where $b=2 \times 10^{6} \mathrm{~T} \mathrm{~m}^{-1}, n_{s}=1 \times 10^{11}$ $\mathrm{cm}^{-2}$, we find $l_{b}=323 \mathrm{~nm}$ and $\omega_{c}=2.47 \times 10^{12} \mathrm{rad} \mathrm{s}^{-1}$.

\section{A. Transverse electron oscillations}

Equation (3) describes an oscillator swinging between two extrema at $\pm \cos (\theta / 2)$ where the velocity cancels. We seek to parametrize the position of this oscillator as a function of time $t$ by integrating Eq. (3). We obtain 


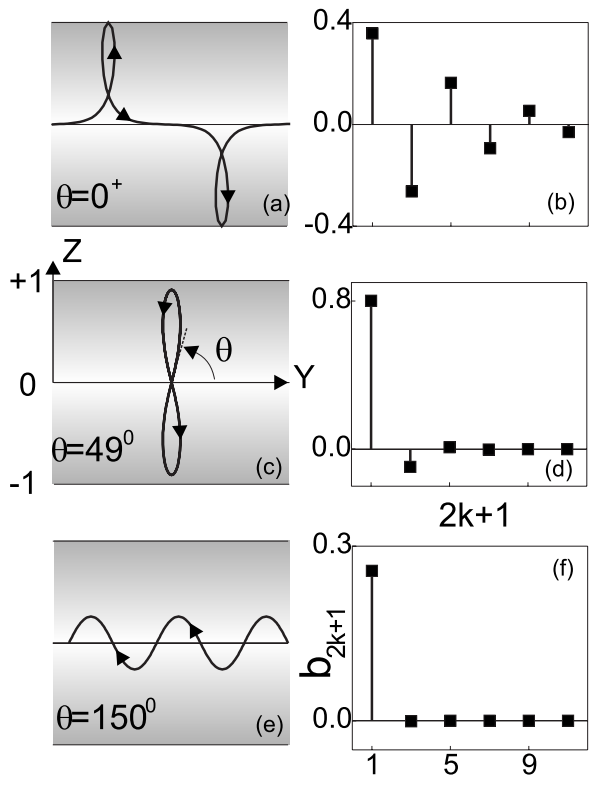

FIG. 2. Semiclassical trajectories of two-dimensional electrons in a magnetic field gradient for three different values of the angle $\theta$ at which the trajectory cuts the center of the channel [(a), (c), and (e)]. Panels (b), (d), and (e) plot the Fourier spectra of the transverse oscillatory motion at the corresponding values of $\theta$.

$$
t=\tau_{b} F(\chi, \theta),
$$

where $F$ is an elliptic integral of the first kind: ${ }^{20}$

$$
F(\chi, \theta)=\int_{0}^{\chi} \frac{d \alpha}{\sqrt{1-\cos ^{2}(\theta / 2) \sin ^{2}(\alpha)}},
$$

and $\chi=\arcsin \sqrt{Z^{2} /\left[\cos ^{2}(\theta / 2)\left(Z^{2}+\sin ^{2}(\theta / 2)\right]\right.}$. The constant of integration assumes that at $t=0$ the particle starts from $(Y, Z)=(0,0)$. A quarter of the period later, the oscillator reaches the apex of the orbit at $Z_{\max }=\cos (\theta / 2)$. By inserting this value into Eq. (4), we obtain the oscillator period as $T$ $=4 \tau_{b} F(\pi / 2, \theta)$. The fundamental frequency of the oscillator follows as $\omega=2 \pi / T$. We then write the position of the oscillator as a Fourier expansion of the fundamental frequency and obtain ${ }^{21}$

$$
\begin{aligned}
Z(t) & =a_{0}+\sum_{i=1}^{\infty}\left(a_{n} \cos (n \omega t)+b_{n} \sin (n \omega t)\right) \\
& =\sum_{k=0}^{\infty} b_{2 k+1} \sin ((2 k+1) \omega t),
\end{aligned}
$$

where

$$
b_{2 k+1}=\frac{4}{(2 k+1) \pi} \int_{0}^{Z_{\max }} d Z \cos \left[(2 k+1) \frac{\pi}{2} \frac{F(\chi, \theta)}{F(\pi / 2, \theta)}\right] .
$$

The oscillators described by Eq. (3) present very interesting properties. Figure 2 shows the three categories of orbits present in the waveguide which we classify according to their drift velocity. Panels (a) and (b) represent orbits with

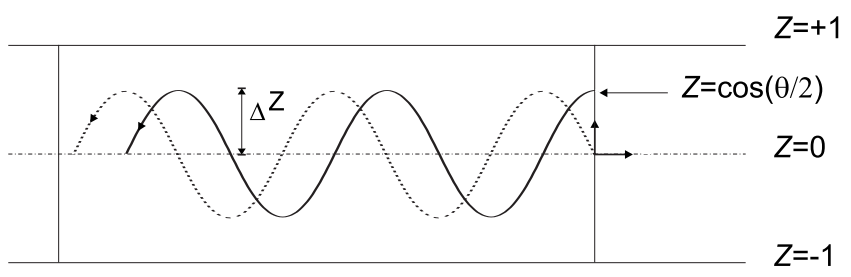

(a)

$\pi / 2<\theta<\pi, \theta>\theta_{\mathrm{c}}$

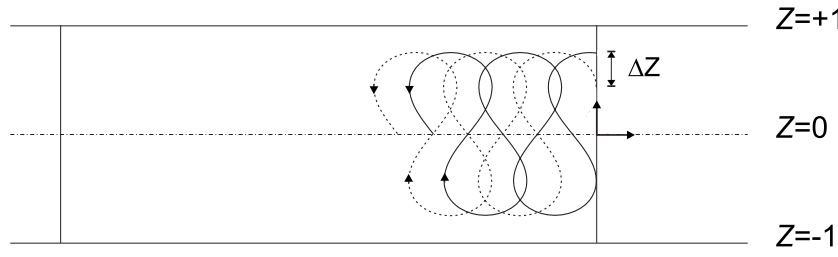

(b)

$$
0<\theta<\pi / 2, \theta>\theta_{c}
$$

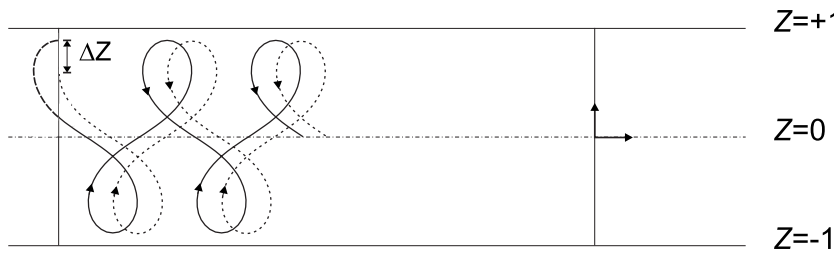

(c)

$$
0<\theta<\pi / 2, \theta<\theta_{\text {c }}
$$

FIG. 3. The range of heights $(\Delta Z)$ from which an electron may enter the waveguide into orbit $\theta$. (a) Shows that orbits cutting the center at a large angle are injected between $Z=0$ and the apex of the orbit at $Z_{\max }=\cos (\theta / 2)$. (b) Shows orbits with intermediate angle $\theta$ which may start with initial velocity transverse to the waveguide. (c) Shows orbits with small $\theta$ that drift in the opposite direction.

small $\theta$ values, positive drift velocity, small oscillator frequency, and large amplitude oscillations. Panels (c) and (d) show "8-shaped" orbits which are stationary. Panels (e) and (f) display orbits with large $\theta$ values, which have a negative drift velocity, fast oscillator frequency and small amplitude. It is clear from this figure that these oscillators cover a range of frequencies when $\theta$ is varied between 0 and $\pi$. A remarkable property is that this frequency range is finite. The upper frequency $\omega_{c}$ corresponds to orbits $\theta=\pi$ which gives $F(\pi / 2, \theta)=\pi / 2$ and $\omega_{c}=\tau_{b}^{-1}$. The lower boundary is $\omega=0$ at $\theta=0$. Mathematically speaking, the elliptic integral in Eq. (5) is not defined for $\theta=0$ or $\pi$. The physical reason is that along the $B_{1}=0$ line, electrons move as free particles independent of the value of the magnetic field gradient.

A second observation relates to the anharmonicity of the oscillators described by Eq. (3). Figure 2 shows that their harmonic content depends on $\theta$ and is particularly rich close to $\theta=0$. The reason is evident from Fig. 2(a) where the approach to the center of the waveguide is asymptotically long. By setting $\theta=0^{+}$in Eq. (3), we find this approach to be exponential,

$$
Z(t)=\frac{2 \exp \left( \pm t / \tau_{b}\right)}{1+4 \exp \left( \pm 2 t / \tau_{b}\right)}
$$


The asymptotic trend is identical at long times independent of whether the argument of the exponential is positive or negative. Interestingly, the time scale of the approach is the characteristic time of the system: $\tau_{b}$. At large values of $t$, $Z(t) \propto \exp \left(-|t| / \tau_{b}\right)$. This causes the oscillator period to diverge and explains why the frequency drops to zero at $\theta=0$. If instead one considers fast oscillators at the other end of the spectrum $(\theta= \pm \pi)$, the amplitude of oscillations becomes vanishingly small. Setting $Z_{\max } \ll 1$ in Eq. (3), the magnetic potential becomes parabolic and takes the familiar form of a harmonic oscillator:

$$
\dot{Z}^{2}+\tau_{b}^{-2} Z^{2}=0 .
$$

One verifies that the fastest oscillators have frequency $\omega_{c}=\tau_{b}^{-1}$. Figure 2(f) shows that the harmonics of the fundamental frequency do not become noticeable until $\theta$ is smaller than $\pi / 2$. We find that it is a sensible approximation to consider oscillators in the interval $[\pi / 2, \pi]$, as harmonic oscillators. Anharmonic oscillators for $\theta<\pi / 2$ will experience a complex periodic magnetic field resulting in satellite resonances to the fundamental spin resonant line. In the reference frame of the oscillating electron, its spin will experience a time dependent magnetic field $B_{1}(t)=b l_{b} Z(t)$.

\section{B. Supply function}

We now calculate the fraction $n(\theta) d \theta$ of the drain-source current injected into open orbits between $\theta$ and $\theta+d \theta$. We make the assumption that the current is carried by magnetic edge states at the center of the waveguide ignoring the magnetoelectric states at its edges. We include in mode $\theta$ all orbits, not only those starting from $Z=0$ at the edge of the waveguide but also those entering the waveguide at finite height, $Z_{0}$. The orbits within mode $\theta$, therefore, connect to the electrodes over a range of heights, comprised between $Z_{\min }$ and $Z_{\max }$, within which the open orbit is accessible to an electron coming from the electrode. $Z_{\min }$ depends on whether the drift velocity is positive or negative and within the latter case whether $\theta$ is larger or smaller than $\pi / 2$. The three cases are depicted in Fig. 3. We calculate $n(\theta)$ by summing the velocities of incoming electrons in the interval $Z_{\min }$ to $Z_{\max }$ and by normalizing by the total number of injected electrons:

$$
n(\theta)=2 \pi \frac{I}{2 e} \frac{\int_{Z_{\min }}^{Z_{\max }} d Z_{0}\left\langle v_{y}\left(\theta, Z_{0}, t\right)\right\rangle}{\int_{0}^{\pi} d \theta \int_{Z_{\min }}^{Z_{\max }} d Z_{0}\left\langle v_{y}\left(\theta, Z_{0}, t\right)\right\rangle} .
$$

The velocities are calculated for one-dimensional states which have a probability $\exp (-t / \tau)$ to remain unscattered at time $t$ :

$$
\left\langle v_{y}\right\rangle=\frac{1}{\tau} \int_{0}^{\infty} d t \exp (-t / \tau) v_{y}\left(\theta, z_{0}, t\right) .
$$

By inserting Eqs. (6) and (7) into Eq. (2), we obtain

$$
\begin{aligned}
\frac{\left\langle v_{y}\right\rangle}{v_{F}}= & \cos \theta-2\left\langle Z^{2}\right\rangle \\
= & \cos \theta-\sum_{k=0}^{\infty} \sum_{k^{\prime}=0}^{\infty} b_{2 k+1} b_{2 k^{\prime}+1}\left[\frac{\cos \left(2\left(k-k^{\prime}\right) \omega t_{0}\right)+2\left(k-k^{\prime}\right) \omega \tau \sin \left(2\left(k-k^{\prime}\right) \omega t_{0}\right)}{1+\left(2\left(k-k^{\prime}\right) \omega \tau\right)^{2}}\right. \\
& \left.-\frac{\cos \left(2\left(k+k^{\prime}+1\right) \omega t_{0}\right)+2\left(k+k^{\prime}+1\right) \omega \tau \sin \left(2\left(k+k^{\prime}+1\right) \omega t_{0}\right)}{1+\left(2\left(k+k^{\prime}+1\right) \omega \tau\right)^{2}}\right]
\end{aligned}
$$

When $\omega \tau \gg 1$, Eq. (10) simplifies to

$$
\frac{\left\langle v_{y}\right\rangle}{v_{F}}=\cos (\theta)-\sum_{k=0}^{\infty} b_{2 k+1}^{2} .
$$

Figure 4(a) shows the range of heights within which an orbit $\theta$ is accessible to an electron of the source. The top of this range is $Z_{\max }$ since no electron can be injected above the apex of the orbit. Orbits between $\pi / 2$ and $\pi$ have a longitudinal velocity which oscillates while keeping a constant negative sign. This allows electrons to be injected from height $Z=0$. Below $\pi / 2$, the longitudinal velocity changes sign at $Z_{\text {min }}=\cos (\theta) / 2$ : it is positive for $Z<Z_{\text {min }}$; however, since electrons incoming into the waveguide have negative velocity, the range of injection heights will be $Z_{\min }<Z_{0}$ $<Z_{\max }$. The vertical line in Fig. 4 corresponds to the change of sign of the electron average drift velocity. This quantity is plotted in Fig. 4(b) for $\tau=\infty$ (dotted curve) and $\tau=1$ ps (solid curve) using Eqs. (12) and (13). When $\theta$ approaches $\pi$, the average drift velocity is close to the Fermi velocity. This enables fast oscillators to carry a large current. Figure 4(c) plots the supply function into individual open orbits. The area under the curve in the $\left[0, \theta_{c}\right]$ (respectively, $\left[\theta_{c}, \pi\right]$ ) interval is unity.

We complete the analysis of the longitudinal motion by calculating the "wavelength" of an open orbit, $\lambda(\theta)$. This is the distance covered in the $y$ direction during one period of the oscillator. Using Eqs. (4) and (13), we obtain 


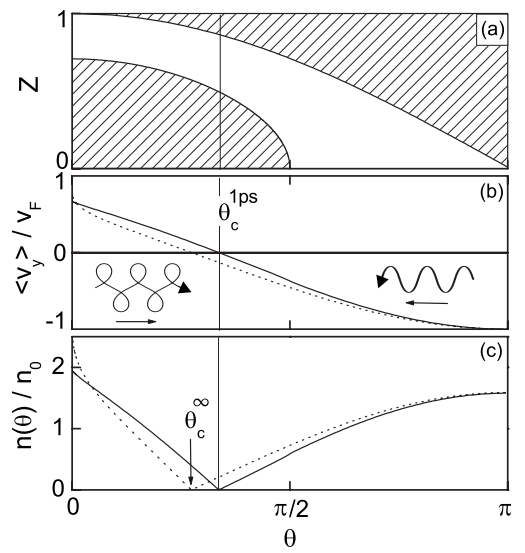

FIG. 4. (a) The clear area represents the range of heights at the edge of the waveguide from which an electron in the source can be injected into orbit $\theta$. (b) Electron drift velocity as a function of $\theta$ for $\tau=1 \mathrm{ps}$ (solid curve) and $\tau=\infty$ (dotted curve). (c) Electron supply function into orbit $\theta$.

$$
\begin{aligned}
\lambda(\theta) & =|Y(t=T)-Y(t=0)| \\
& =2 l_{b} F(\pi / 2, \theta)\left|\cos (\theta)-\sum_{k=0}^{\infty} b_{2 k+1}^{2}\right| .
\end{aligned}
$$

It follows that the wavelength of the fastest oscillators is $\lambda(\pi)=\pi l_{b}$. It is useful to note that despite the vanishing amplitude of oscillations, the wavelength remains finite and relatively large. The wavelength represents the minimum distance over which spin coherence must be conserved for the spin to experience a periodic magnetic field. The magnetic length must therefore be as small as possible for observing spin Rabi oscillations.

Having described the dynamics of the electron oscillator, we now calculate the dynamics of its spin and its interaction with the electromagnetic field.

\section{RADIATIVE EMISSION RATE}

In the magnetic waveguide, the magnetic field $B_{0}$ splits the spin degenerate states into a ground and an excited state $\{|g\rangle,|e\rangle\}$. In addition, the spin senses the ac magnetic field $B_{1}(t)$ arising from oscillations in the magnetic field gradient. Electron spin resonance takes place when both frequencies match, namely, at $\omega=\omega_{0}$. In addition, the spin interacts with the magnetic component of the electromagnetic field, $B_{\text {phot }}$, which accounts for radiative losses by spontaneous emission. Since the oscillating magnetic field $B_{1}$ does not increment or decrement the number of photons in the system, we solve the radiative problem by considering the two quantum states $\{|g, 0\rangle$ and $|e, 0\rangle\}$ dressed by the ground state of the field (Fig. 5). The field is here assumed to be empty as photons spontaneously emitted escape to infinity. Those two quantum states interact with the electromagnetic continuum $|g, \vec{k}, p\rangle$, where $\vec{k}$ and $p$ are the photon wave vector and polarization. The Hamiltonian of the system is

$$
H=H_{0}+V_{B}(t)+V_{p h o t}(t),
$$

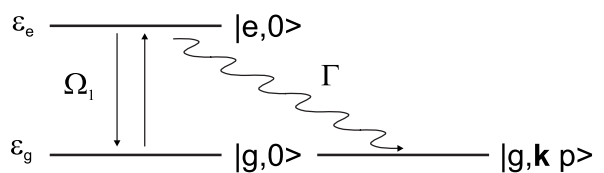

FIG. 5. Energy diagram of spin resonance fluorescence. The two spin states $|g\rangle$ and $|e\rangle$ are coupled via the ac magnetic field arising from electron oscillations in the magnetic field gradient. At resonance Rabi, oscillations occur at frequency $\Omega_{1}$ without the creation or annihilation of a photon- $|g, 0\rangle$ and $|e, 0\rangle$ have the same photon number. The excited state decays by spontaneously emitting photons with random polarization and direction at rate $\Gamma$.

$$
\begin{gathered}
H_{0}=-\gamma S_{z} B_{0}, \\
V_{B}(t)=-\gamma S_{x} b l_{b} Z(t), \\
V_{p h o t}(t)=-\gamma \vec{S} \cdot \vec{B}_{p h o t}(t) .
\end{gathered}
$$

We aim to find the time evolution of the quantum system and first calculate the Green's operator $G\left(z_{ \pm}\right)=\left[z_{ \pm}-H\right]^{-1}$ in the basis of states $\{|g, 0\rangle$ and $|e, 0\rangle\}$. We have used $\gamma$ $=-e /\left(2 m_{0}\right)$ as the spin gyromagnetic ratio and $z_{ \pm}=E \pm i \eta$, where $E$ is the energy. The Green's matrix in the two level subspace is

$$
\left[\begin{array}{cc}
G_{g g} & G_{g e} \\
G_{e g} & G_{e e}
\end{array}\right]=\frac{1}{\Delta}\left[\begin{array}{cc}
z-E_{e}-\Sigma_{e e} & \Sigma_{g e} \\
\Sigma_{e g} & z-E_{g}-\Sigma_{g g}
\end{array}\right],
$$

where

$$
\Delta=\left(z-E_{g}-\Sigma_{g g}\right)\left(z-E_{e}-\Sigma_{e e}\right)-\Sigma_{e g} \Sigma_{g e} .
$$

It is sufficient to calculate the self-energies $\Sigma_{g g}, \Sigma_{e e}, \Sigma_{g e}$ to first order in the magnetodipole interaction since the coupling to the electromagnetic field is weak.

$$
\begin{gathered}
\Sigma_{e e}=\hbar \Delta_{e}-i \frac{\hbar \Gamma_{e}}{2}, \\
\Delta_{e}=\frac{1}{\hbar} \wp\left\{\sum_{k, p} \frac{\left|\left\langle e, 0\left|V_{p h o t}\right| g, \vec{k}, p\right\rangle\right|^{2}}{E-E_{g}-\hbar \omega}\right\}, \\
\Gamma_{e}=\frac{2 \pi}{\hbar} \sum_{k, p}\left|\left\langle e, 0\left|V_{p h o t}\right| g, \vec{k}, p\right\rangle\right|^{2} \delta\left(E-E_{g}-\hbar \omega\right) .
\end{gathered}
$$

For the ground state, one obtains $\Sigma_{g g}=\hbar \Delta_{g}$. Radiative decay from the ground state is forbidden by conservation of energy and consequently $\Gamma_{g}=0$. The only nonzero term is the real energy shift:

$$
\Delta_{g}=\frac{1}{\hbar} \wp\left\{\sum_{k, p} \frac{\left|\left\langle g, 0\left|V_{p h o t}\right| g, \vec{k}, p\right\rangle\right|^{2}}{E-E_{g}-\hbar \omega}\right\} .
$$

The off-diagonal self-energy $\Sigma_{g e}$ features different spin states with the same photon number. These are coupled via the oscillatory field $B_{1}(t)$. We have 


$$
\Sigma_{g e}=\left\langle g, 0\left|V_{B}\right| e, 0\right\rangle=-\gamma b l_{b} b_{2 k+1}\left\langle g, 0\left|S_{x}\right| e, 0\right\rangle=-\frac{\hbar \Omega_{1}}{2},
$$

where $\Omega_{1}=\gamma b l_{b} b_{2 k+1}$ is the Rabi frequency of mode $2 k+1$. This interaction hybridizes the quantum states, shifting their energy levels and modifying their lifetimes. To obtain the eigenenergies, we solve the following effective Hamiltonian:

$$
H_{e f f}=\left[\begin{array}{cc}
\hat{E}_{g} & \Sigma_{g e} \\
\Sigma_{e g} & \hat{E}_{e}-i \hbar \frac{\Gamma_{e}}{2}
\end{array}\right],
$$

where we have set

$$
\begin{aligned}
& \hat{E}_{g}=E_{g}-\Delta_{g}, \\
& \hat{E}_{e}=E_{e}-\Delta_{e} .
\end{aligned}
$$

The effect of switching on the interaction between the spin and the electromagnetic field is to lower the spin energy levels by an amount equal to the self-energy. This results in the spin resonance emission line being shifted by $\Delta_{g}-\Delta_{e}$.

We calculate the rate of spontaneous decay from the excited state using the Fermi golden rule in Eq. (23). For this, we assume that the electromagnetic modes within the semiconductor have a three-dimensional density of states and the velocity of light in the semiconductor is $c / \sqrt{\epsilon_{r}}$, where $\epsilon_{r}$ is the relative dielectric constant. We insert in Eq. (18) the magnetic field component of the electromagnetic field: ${ }^{4}$

$$
\vec{B}_{\text {phot }}=\frac{i}{c} \sqrt{\frac{\hbar \omega}{2 \epsilon_{r} \epsilon_{0} L^{3}}}\left[a \vec{n} e^{i \vec{k} \vec{r}}-a^{\dagger} \vec{n} e^{-i \vec{k} \vec{r}}\right],
$$

where $a$ and $a^{+}$are the creation and annihilation operators of a photon of energy $\hbar \omega, \epsilon_{0}$ is the dielectric constant of vacuum, $L^{3}$ is the volume of the field, and $\vec{n}$ is the unit vector aligned with the direction of the magnetic polarization. After integration over the final states in Eq. (23), we obtain

$$
\begin{aligned}
\Gamma_{e} & =\frac{2 \pi}{\hbar} \sum_{a} \sum_{\vec{k}, p}\left|\left\langle a, \vec{k} p\left|V_{p h o t}\right| b, 0\right\rangle\right|^{2} \delta\left(E_{b}-E_{a}-\hbar \omega\right) \\
& =\frac{1}{3} \frac{\left(g \mu_{B}\right)^{2} k^{3}}{c e^{2}} \alpha,
\end{aligned}
$$

where $\mu_{B}$ is the Bohr magneton, $k=\sqrt{\epsilon_{r}} \omega / c$ is the photon wave vector, and $\alpha=e^{2} /\left(4 \pi \epsilon_{0} \hbar c\right) \approx 1 / 137$ is the fine structure constant. The electromagnetic field couples to the $S_{z}$ component of spin only. The magnetodipole radiative rate is quite weak since even at the top frequency of spin oscillators, $500 \mathrm{GHz}$, one has $\Gamma=3.92 \times 10^{-4} \mathrm{~s}^{-1}$. In a typical 2DEG, one has of the order of $N=10^{10}$ spins oscillating at the same frequency and possibly in phase by interacting through the electromagnetic field that they emit. Under these conditions, the radiative scattering rate increases to $\Gamma_{\mathrm{SR}}$ $=N \Gamma$ through super-radiant emission. ${ }^{22-27}$ We will develop this idea when calculating microwave emission spectra in the following paper. ${ }^{28}$

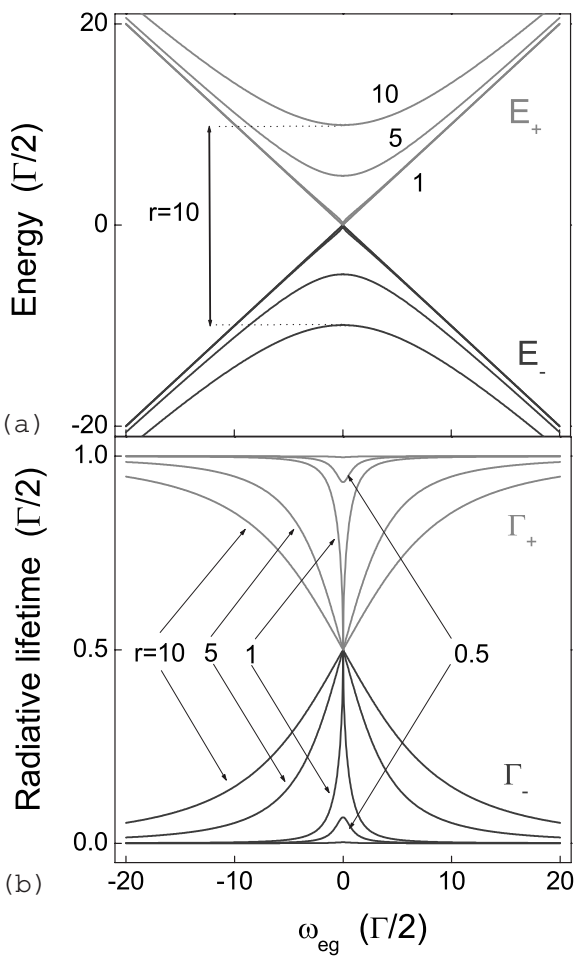

FIG. 6. Energies (a) and lifetimes (b) of two metastable spin states $|g\rangle$ and $|e\rangle$ plotted as a function of the detuning frequency (see text). The spin states interact through an ac magnetic field $\Omega_{1}$ and couple to the electromagnetic continuum at a rate $\Gamma$. The curves are given for different values of the ratio $r=\Omega_{1} /(\Gamma / 2)$. The origin in energy was arbitrarily set at $\hat{E}_{0}$.

The diagonalization of Eq. (26) gives the following energy eigenvalues:

$$
E_{ \pm}=\hat{E}_{0} \mp \frac{\hbar \Gamma}{4} \sqrt{\chi \rho}-i \frac{\hbar \Gamma}{4}(1 \mp \sqrt{\chi / \rho}) .
$$

We have used $\hat{E}_{0}=\left(\hat{E}_{g}+\hat{E}_{e}\right) / 2, \omega_{e g}=\omega-\left(\hat{E}_{e}-\hat{E}_{g}\right) / \hbar$, and

$$
\begin{gathered}
\chi=\frac{\left|\omega_{e g}\right|}{\Gamma / 2}, \\
\rho=\frac{\delta}{-\operatorname{sgn}\left(\Omega^{2}\right)+\sqrt{1+\delta^{2}}}, \\
\Omega^{2}=\Omega_{1}^{2}+\omega_{e g}^{2}-\frac{\Gamma^{2}}{4}, \\
\delta=\frac{\Gamma\left|\omega_{e g}\right|}{\left|\Omega^{2}\right|} .
\end{gathered}
$$

Figure 6(a) plots the real part of $E_{ \pm}$which shows the hybridization of the dressed states near resonance. Figure 6(b) shows the imaginary part of the energy which represents their rate of decay. When the oscillator is resonant with the transition frequency, the eigenstates form an even mix of spin up and spin down states. The explanation is that during Rabi oscillations the spin spends the same amount of time in 
the ground state and the excited state. The energy gap, $\hbar \Omega_{1}$, gives the rate at which these oscillations take place. In the lower panel, the hybridized states radiate at the same rate of $\Gamma / 4$. This is because state $|e, 0\rangle$ has an equal weight in both hybrid states. Further from resonance, the radiative lifetimes of hybridized states tend to the radiative lifetimes of states $|g, 0\rangle$ and $|e, 0\rangle$. If one reduces the Rabi coupling below the rate of spontaneous decay, hybridization disappears even at resonance. This is because the coupling between states $|g, 0\rangle$ and $|e, 0\rangle$ needs time to establish itself which does not happen if the state decays too rapidly. Returning to the magnetic waveguide, those spin oscillators which have small amplitudes will couple more strongly to the electromagnetic field. This is because they experience a vanishingly small magnetic field $B_{1}(t)$ and therefore satisfy the condition $\Omega_{1}$ $<\Gamma / 2$.

In Fig. 1, the spin starts from the source electrode initially prepared either in state $|g, 0\rangle$ or $|e, 0\rangle$. We now calculate its evolution toward the excited state $|e, 0\rangle$ as a function of time. The probability of spontaneous emission between $t$ and $t$ $+d t$ is the probability of occupancy of the excited state times the rate of spontaneous decay. We obtain the probability of evolution toward the excited state by contour integration of matrix elements in Eq. (19)

$$
U(t)=\frac{1}{2 \pi i} \int_{C} d z e^{-i z t / \hbar} G(z) .
$$

For the spin initially prepared in state $|g, 0\rangle$, we obtain

$$
\begin{aligned}
U_{e g}(t) & =\frac{-\hbar \Omega_{1} / 2\left[e^{-i E_{+} t / \hbar}-e^{-i E_{-} t / \hbar}\right]}{E_{+}-E_{-}} \\
& =\frac{i \Omega_{1}}{(\Gamma / 2)} \frac{\sin \left[\chi\left(\rho-i \rho^{-1}\right) \frac{\Gamma t}{4}\right]}{\chi\left(\rho-i \rho^{-1}\right)} e^{-i \hat{E}_{0} t / \hbar} e^{-\Gamma t / 4} .
\end{aligned}
$$

A similar calculation for a spin injected from state $|e, 0\rangle$ gives

$$
\begin{aligned}
U_{e e}(t)= & \frac{\left(E_{+}-\widetilde{E}_{g}\right) e^{-i E_{+} t / \hbar}-\left(E_{-}-\widetilde{E}_{g}\right) e^{-i E_{-} t / \hbar}}{E_{+}-E_{-}} \\
= & {\left[\cos \left[\chi\left(\rho-i \rho^{-1}\right) \frac{\Gamma t}{4}\right]\right.} \\
& \left.-(1+i \chi) \frac{\sin \left[\chi\left(\rho-i \rho^{-1}\right) \frac{\Gamma t}{4}\right]}{\chi\left(\rho-i \rho^{-1}\right)}\right] e^{-i \hat{E}_{0} t / \hbar} e^{-\Gamma t / 4} .
\end{aligned}
$$

The probability that a photon is emitted between time $t$ and $t+d t$ is given by $\Gamma\left|U_{e g}(t)\right|^{2}$ if the spin starts from state $|g, 0\rangle$ and $\Gamma\left|U_{e e}(t)\right|^{2}$ if it starts from $|g, 0\rangle$. The probability from $|g, 0\rangle$ to $|e, 0\rangle$ is easily calculated as

$$
\Gamma\left|U_{e g}(t)\right|^{2}=\frac{\Gamma}{2} \frac{\Omega_{1}^{2}}{\lambda_{1}^{2}+\lambda_{2}^{2}}\left(\cosh \left(\lambda_{2} t\right)-\cos \left(\lambda_{1} t\right)\right) e^{-\Gamma t / 2},
$$

where $\lambda_{1}=(\Gamma / 2) \sqrt{\chi \rho}$ and $\lambda_{2}=(\Gamma / 2) \sqrt{\chi / \rho}$. One also verifies that

$$
\int_{0}^{\infty} d t \Gamma\left|U_{e g}(t)\right|^{2}=1
$$

In order to describe the properties of single photon emission which are contained in Eqs. (37) and (39), we start by separately discussing the regime of strong coupling to the electromagnetic field $\left(\Gamma \gg \Omega_{1}\right)$ and the weak coupling regime $\left(\Gamma \ll \Omega_{1}\right)$. In the strong coupling regime, $\lambda_{1}$ and $\lambda_{2}$ approximate to

$$
\begin{aligned}
\lambda_{1} & =\omega_{e g}(1+\alpha), \\
\lambda_{2} & =\frac{\Gamma}{2}(1-\alpha),
\end{aligned}
$$

where $\alpha=\frac{\Omega_{1}^{2} / 2}{\omega_{e g}^{2}+\Gamma^{2 / 4}} \ll 1$. At resonance, $\lambda_{1}$ vanishes with the consequence that in Eq. (39), the frequency of spin flip oscillations cancels. Inserting Eqs. (41) and (42) in Eq. (39) gives at long times an emission probability which decreases very slowly: $\Gamma\left|U_{e g}(t)\right|^{2} \approx\left(\Omega_{1}^{2} /(\Gamma / 2)\right) \exp (-\alpha \Gamma t / 2)$. The spin injected from the excited state will initially decay rapidly at a rate $\Gamma / 2$ and then at a slower rate $\alpha \Gamma / 2$. By contrast, the spin initially prepared in the ground state cannot decay radiatively. This is shown in Fig. 7(a) where the emission probability is small even at resonance. Figure 7(b) shows the exponential decay from a spin prepared in the excited state.

In the weak coupling regime $\Omega_{1} \gg \Gamma / 2, \lambda_{1}$ and $\lambda_{2}$ approximate to

$$
\begin{gathered}
\lambda_{1}=\Omega, \\
\lambda_{2}=\frac{\Gamma}{2} \frac{\left|\omega_{e g}\right|}{\Omega} .
\end{gathered}
$$

This regime corresponds to the development of Rabi oscillations seen in Fig. 8 as the electron progresses into the waveguide. In Fig. 8(a), the spin initially in state $|g, 0\rangle$ has a significant probability of flipping into state $|e, 0\rangle$ at the end of the first half period. Rabi oscillations therefore enable a spin injected into the nonradiative ground state to emit a photon at well defined time intervals. It is notable that oscillations are less damped at resonance showing that spontaneous emission will take place at distances well beyond the exponential decay length. In Fig. 8(b), the spin is injected from the excited state. Its probability in the excited state decays while oscillating. The resonant beats die out at a slower rate than the nonresonant beats.

We have therefore investigated the conditions by which a spin prepared parallel or antiparallel to $B_{0}$ proceeds into the waveguide and radiates while performing coherent oscillations. We have discussed the strong and the weak coupling regime. The strong coupling regime corresponds to fast oscillators at the edge of the dispersion curve $(\theta \approx \pi)$, whereas the weak coupling regime corresponds to the slower oscilla- 


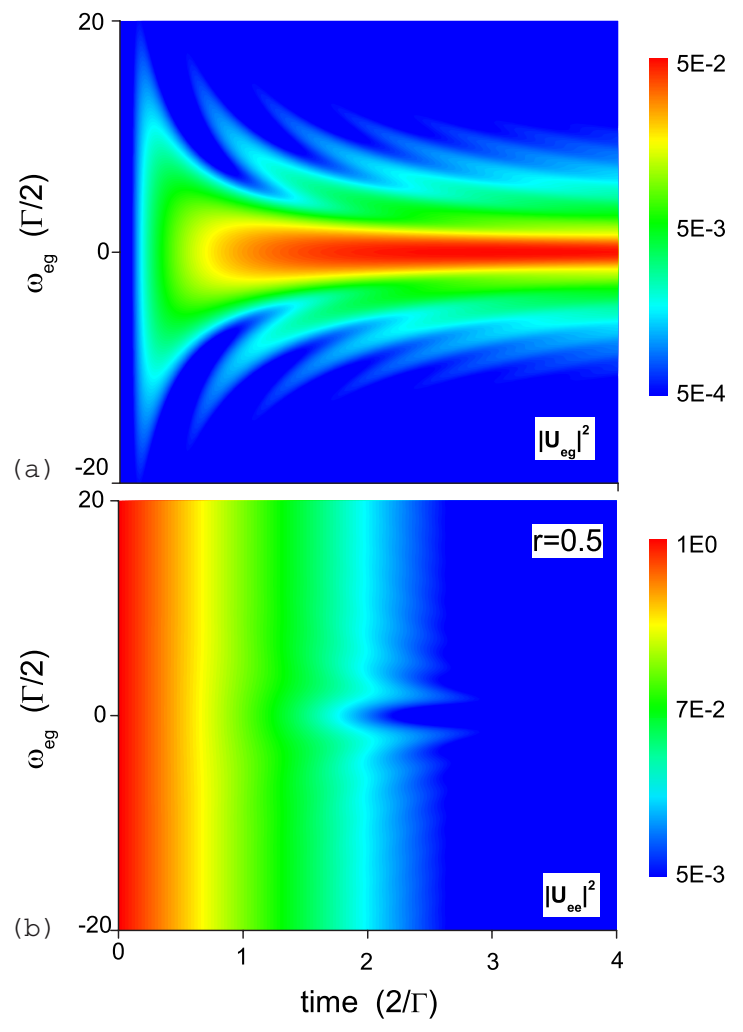

FIG. 7. (Color online) Probability of emission of one photon as a function of the time spent by the electron oscillating in the waveguide. In the strong coupling regime shown here $(r=0.5)$, radiative decay occurs on the scale of $\Gamma / 2$ for electrons entering the waveguide in the spin down state $\left[r \equiv \Omega_{1} /(\Gamma / 2)\right]$. Radiative decay is quasi-nonexistent from electrons prepared in the spin up state.

tors at its center which experience a large amplitude ac magnetic field. We will now be using the above results to calculate the power generated by a sequence of radiative decays emanating from an oscillator as it performs Rabi oscillations.

\section{PHOTON ANTIBUNCHING AND RADIATIVE CASCADE}

We define $p_{g}^{(1)}(t)$ as the probability that a spin injected from the ground state has emitted one photon and one photon only after a time $t$ in the waveguide. This is expressed as

$$
p_{g}^{(1)}(t)=\int_{0}^{t} d t_{1} \Gamma\left|U_{e g}\left(t_{1}\right)\right|^{2}
$$

During the same time interval, the oscillator may have emitted a first photon at time $t_{1}\left(t_{1}<t\right)$ and a second one in the remainder of the interval. The probability of this two photon process is the product of the one photon probabilities summed over all possible times for the emission of the first photon. After the emission of the first photon, coherent Rabi oscillations will always restart from $g$. For the spin initially in the ground state, the two photon probability is

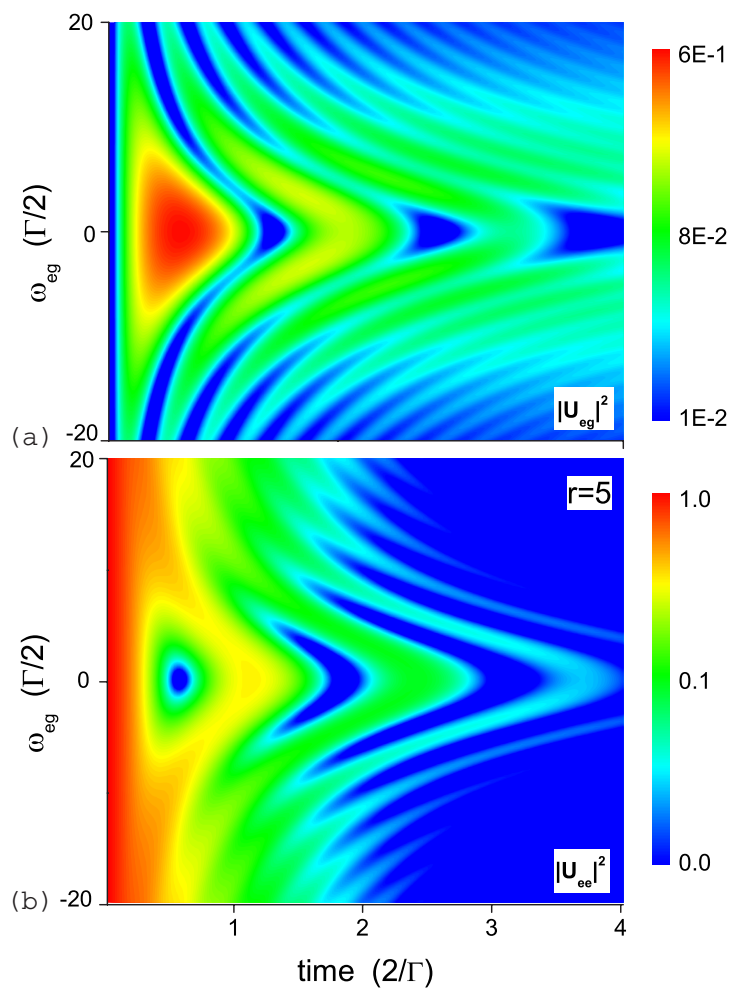

FIG. 8. (Color online) Probability of emission of one photon as a function of time spent by the oscillator in the waveguide. In (a) [(b)], the electron enters the waveguide in the spin state of low (high) energy. In the weak coupling regime shown here $(r=5)$, Rabi oscillations occur and prolong radiative emission beyond $2 / \Gamma$.

$$
p_{g}^{(2)}(t)=\int_{0}^{t} d t_{1} \int_{0}^{t_{1}} d t_{2} \Gamma^{2}\left|U_{e g}\left(t_{1}-t_{2}\right)\right|^{2}\left|U_{e g}\left(t_{2}\right)\right|^{2} .
$$

In the same way, the probability of a three photon event is

$$
\begin{aligned}
p_{g}^{(3)}(t)= & \int_{0}^{t} d t_{1} \int_{0}^{t_{1}} d t_{2}\left|U_{e g}\left(t_{1}-t_{2}\right)\right|^{2} \\
& \times \int_{0}^{t_{2}} d t_{3} \Gamma^{3}\left|U_{e g}\left(t_{2}-t_{3}\right)\right|^{2}\left|U_{e g}\left(t_{3}\right)\right|^{2} .
\end{aligned}
$$

For an electron starting from $|e, 0\rangle$, we have similarly

$$
p_{e}^{(1)}(t)=\int_{0}^{t} d t_{1} \Gamma\left|U_{e e}\left(t_{1}\right)\right|^{2} .
$$

For multiple photon emission starting from $|e, 0\rangle$, the emission probability is given by $U_{e e}$ during the first time interval and by $U_{g e}$ in the following ones. We therefore write

$$
p_{e}^{(2)}(t)=\int_{0}^{t} d t_{1} \int_{0}^{t_{1}} d t_{2} \Gamma^{2}\left|U_{e g}\left(t_{1}-t_{2}\right)\right|^{2}\left|U_{e e}\left(t_{2}\right)\right|^{2}
$$

and

$$
\begin{aligned}
p_{e}^{(3)}(t)= & \int_{0}^{t} d t_{1} \int_{0}^{t_{1}} d t_{2}\left|U_{e g}\left(t_{1}-t_{2}\right)\right|^{2} \int_{0}^{t_{2}} d t_{3} \Gamma^{3} \\
& \times\left|U_{e g}\left(t_{2}-t_{3}\right)\right|^{2}\left|U_{e e}\left(t_{3}\right)\right|^{2}
\end{aligned}
$$




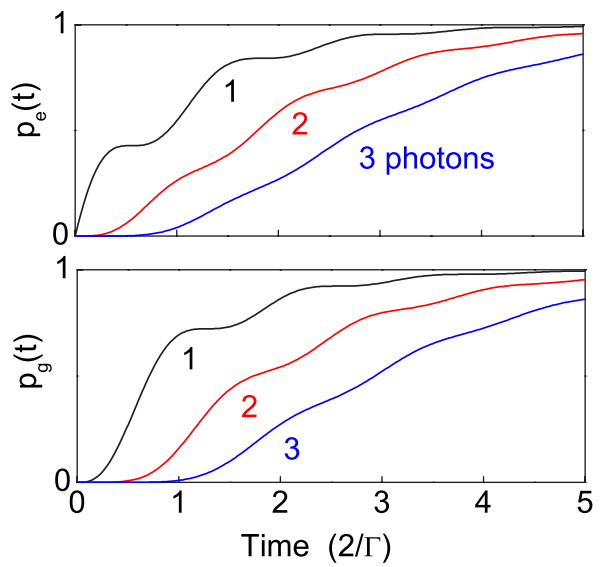

FIG. 9. (Color online) Probability for the cascade emission of 1, 2 , or 3 photons after a given time in the weak coupling regime $(r$ $=5)$.

Figure 9 plots the probability that a single photon, or two photons, or three photons have been emitted after a certain time. These probabilities were obtained by integrating numerically Eqs. (30)-(36) when the frequency of the oscillator is resonant with the frequency of the transition in the weak coupling regime, $\Omega_{1}=5(\Gamma / 2)$. The top (bottom) graph plots the three probabilities for a spin oscillating from state $e(g)$. Naturally, $p_{e}^{(1)}$ rises rapidly as the electron decays from the metastable initial state. The onset of the $p_{e}^{(2)}$ and $p_{e}^{(3)}$ probabilities is seen to take place at later times as these events, respectively, require the one and two photon processes to have established themselves prior to the emission of the next photon. In addition, the spin restarts from the ground state after each radiative decay and requires a spin flip before it can radiate again. This delays the emission of a photon by half the Rabi period, an effect which is observed in the lower panel. In the lower panel, all cascading events starting from $g$ are delayed relative to events starting from $e$. We note that the probabilities oscillate with time. This is because the time intervals between consecutive emissions are correlated even if radiative events occur randomly. These periodic bursts of radiation are predicted to occur at regular distances along the waveguide giving photon antibunching ${ }^{29-31}$ delocalized in space. In real structures, one will achieve $r \gg 1$ giving very fast oscillations with periodicity on the scale of the micrometer. For this reason, photon antibunching ought to be observed by tuning the wavelength of open orbits in the waveguide $\lambda(\pi)=\pi l_{b}$. This may be done by applying a gate voltage to change $l_{b}$. It is noteworthy that the oscillations of $p_{g}$ and $p_{e}$ are out of phase which doubles the frequency of the fluorescence signal. Over small time scales, the probability of emission depends on the initial spin state. This dependence rapidly disappears as time increases until at longer times, the probabilities $p_{e}^{(i)}$ and $p_{g}^{(i)}$ are no longer distinguishable. As a result, the fluorescence signal in a short magnetic waveguide will depend on the spin polarization. By contrast, the fluorescence signal of the longer waveguides is independent of the initial spin polarization because of the loss of spin coherence in the radiative cascade.

The fluorescence power emitted by the spin oscillator $\theta$ at time $t$ is the photon energy multiplied by the electron injec- tion rate $n(\theta)$. This is weighted with the number of photons $i$ released by the cascade, and the probability of the cascade occurring at time $t$. The latter includes probabilities $p_{g}$ and $p_{e}$ weighted by the spin polarization of the incoming current $n_{g}$ and $n_{e}$. Summing over all radiative sequences, we obtain

$$
P_{k}\left(\omega_{e g}, t\right)=n(\theta)(2 k+1) \hbar \omega \sum_{i=1}^{\infty} i\left[n_{g} p_{g}^{(i)}(t)+n_{e} p_{e}^{(i)}(t)\right]
$$

which is the fluorescence emanating from harmonic $k$. Substituting Eqs. (45)-(50) in the series, we obtain

$$
\begin{aligned}
P_{k}\left(\omega_{e g}, t\right)= & n(\theta)(2 k+1) \hbar \omega \\
& \times \int_{0}^{t} d\left(\Gamma t_{1}\right)\left\{f\left(t_{1}\right)+2 \int_{0}^{t_{1}} d t_{2} K\left(t_{1}, t_{2}\right) f\left(t_{2}\right)\right. \\
& \left.+3 \int_{0}^{t_{1}} d t_{2} K\left(t_{1}, t_{2}\right) \int_{0}^{t_{2}} d t_{3} K\left(t_{2}, t_{3}\right) f\left(t_{3}\right)+\ldots\right\},
\end{aligned}
$$

where the initial conditions are included in the injection function:

$$
f(t)=n_{g}\left|U_{e g}(t)\right|^{2}+n_{e}\left|U_{e e}(t)\right|^{2} .
$$

We also define the kernel of the radiative cascade:

$$
K\left(t, t_{1}\right)=\Gamma\left|U_{e g}\left(t-t_{1}\right)\right|^{2},
$$

which describes the coherent evolution of the spin between two radiative events taking place at $t_{1}$ and $t\left(t>t_{1}\right)$. We obtain an exact solution for the infinite sum in Eq. (52). We first define the function $F(t)$,

$$
\begin{aligned}
F(t)= & f(t)+\int_{0}^{t} d t_{1} K\left(t, t_{1}\right) f\left(t_{1}\right) \\
& +\int_{0}^{t} d t_{1} \int_{0}^{t_{1}} d t_{2} K\left(t, t_{1}\right) K\left(t_{1}, t_{2}\right) f\left(t_{2}\right)+\ldots,
\end{aligned}
$$

which is a solution of Volterra's equation,

$$
F(t)=f(t)+\int_{0}^{t} d t_{1} K\left(t, t_{1}\right) F\left(t_{1}\right) .
$$

Equation (52) then becomes

$$
\begin{aligned}
P_{k}\left(\omega_{e g}, t\right)= & n(\theta)(2 k+1) \hbar \omega \\
& \times \int_{0}^{t} d\left(\Gamma t_{1}\right)\left\{F\left(t_{1}\right)+\int_{0}^{t_{1}} d t_{2} K\left(t_{1}, t_{2}\right) F\left(t_{2}\right)\right. \\
& \left.+\int_{0}^{t_{1}} d t_{2} \int_{0}^{t_{2}} d t_{3} K\left(t_{1}, t_{2}\right) K\left(t_{2}, t_{3}\right) F\left(t_{3}\right)+\ldots\right\},
\end{aligned}
$$

where the supply function has been replaced by the new function defined in Eq. (56). The exact solution of Eq. (57) to all orders is obtained by introducing a second function $G(t)$ which satisfies 


$$
G(t)=F(t)+\int_{0}^{t} d t_{1} K\left(t, t_{1}\right) G\left(t_{1}\right)
$$

Hence, the microwave power in Eq. (52) becomes

$$
P_{k}\left(\omega_{e g}, t\right)=n(\theta)(2 k+1) \hbar \omega \int_{0}^{t} d\left(\Gamma t_{1}\right) G\left(t_{1}\right) .
$$

This is an exact result that allows to calculate the fluorescence power to all orders of the radiative cascade. $G(t)$ may be calculated numerically ${ }^{32}$ by solving the system of integral equations, Eqs. (53), (54), (56), and (58). This function is then integrated over the dwell time in the waveguide to give the power output by harmonic $k$. Equation (59) contains the implicit dependence on the length of the waveguide. Long waveguides, such that $\Gamma t>1$, will give a more intense fluorescence signal as they allow longer interaction of the oscillator with the field.

To calculate the power radiated by individual oscillators parametrized by $\theta$, one sums the contribution from its harmonics

$$
P\left(\omega(\theta), \omega_{0}, t\right)=\sum_{k=0}^{\infty} P_{k}\left(\omega_{e g}, t\right)
$$

The fluorescence power emanating from the waveguide is subsequently obtained by integrating over $\theta$,

$$
\left\langle P\left(\omega_{0}, t\right)\right\rangle=\int_{0}^{\pi} d \theta P\left(\omega(\theta), \omega_{0}, t\right) .
$$

\section{DISCUSSION}

We have derived an exact theory of electrically induced spin resonance fluorescence which integrates all parameters of the system. Because cascade emission consists of randomly spaced spontaneous decays, the radiation emitted is mostly incoherent. This is if one includes antibunching and super-radiant, effects. It is well known that the coupling of coherent oscillators enhances the radiated power by a factor of $N^{2}$. One readily verifies this dependence here by substituting the super-radiant rate $\Gamma_{S R}=N \Gamma$ in Eq. (59) or Eq. (64) and by writing explicitly that the electron injection rate $n(\theta)=N I / e$ is the number of resonating electrons inside the waveguide upon the electron dwell in the waveguide. This gives $P \propto N^{2}$.

Another way to enhance coherence is to place spin oscillators in an electromagnetic cavity. ${ }^{4,22}$ This would require modifying the theory in the following ways. On the one hand, the rate of spontaneous decay has to be recalculated by summing over the discrete density of final photon states. The emitted intensity will be enhanced at frequencies of the cavity modes. On the other hand, photons reflected by the cavity walls increase the number of photons in the cavity to a finite number. Spontaneous decay will subsequently occur across a ladder of dressed states from $|e, N\rangle$ to $|g, N+1\rangle,|e, N-1\rangle$ to $|g, N\rangle$, etc. enhancing the intensity of emission. A photon bath with the correct wavelength will also enhance coherence through stimulated emission. It is, however, quite unlikely that Maser action would be observed because the radiative cascade does not support population inversion. A photon bath is finally expected to split the spin resonance line into triplet lines due to the radiative cascade between dressed spin states. ${ }^{3,4}$

The effect of momentum scattering in the waveguide is expected to have no effect on the fluorescence as long as scattering occurs between spin oscillators. If an electron scatters out of the waveguide toward nonmagnetic regions, the lifetime of the relevant spin oscillators decreases according to Eq. (11). This problem becomes similar to spin resonance in a metastable state such as the conduction band of semiconductors ${ }^{7}$ or the $p$ orbital of the hydrogen atom. ${ }^{2}$ If we call, $\gamma=\tau^{-1}$, the momentum scattering rate, the new radiative probability calculated according to Eq. (45) becomes

$$
p_{g}^{(1)}=\int_{0}^{\infty} d t \Gamma\left|U_{e g}(t)\right|^{2} \exp (-\gamma t) .
$$

In the regime of Rabi oscillations $\Omega_{1} \gg \Gamma$, this probability becomes

$$
p_{g}^{(1)}=\frac{\Gamma}{2 \gamma} \frac{\Omega_{1}^{2}}{\gamma^{2}+\Omega_{1}^{2}+\omega_{e g}^{2}} .
$$

The power radiated is

$$
P=n \hbar \omega \frac{\Gamma}{2 \gamma} \sum_{k=0}^{\infty}(2 k+1) b_{2 k+1}^{2} \frac{\Omega_{1}^{2}}{\gamma^{2}+\Omega_{1}^{2}+\left(\omega_{0}-(2 k+1) \omega\right)^{2}},
$$

which, apart from the $\Gamma /(2 \gamma)$ prefactor, is the result that one obtains by considering the spin as a classical magnetic dipole. ${ }^{21}$ If, however, momentum scattering occurs within the bundle of open orbits, the effect on the microwave spectrum will be small. Such transitions create a hole at one point of the Fermi surface and fill the final state with an excess electron. The resulting change imbalance creates an electric field which redistributes charge to screen the hole. Based on this argument, we conclude that as long as electrons do not scatter out of the bundle of open orbits, the microwave spectrum will not be affected by elastic scattering.

\section{CONCLUSION}

In summary, we have derived an exact quantum electrodynamic theory of electrically induced spin resonance fluorescence in magnetoelectric potentials which allows calculating the microwave power emitted by spin oscillators. The theory obtains the parameters of experimental structures. It improves over our earlier work ${ }^{21}$ by explicitly integrating the spin polarization of the injected current, the length of the waveguide, and magnetodipole coupling.

\section{ACKNOWLEDGMENTS}

We acknowledge the support of EPSRC (UK) and the Flemish Science Foundation (FWO-VI). 
*a.r.nogaret@bath.ac.uk

${ }^{1}$ H. Kimble and L. Mandel, Phys. Rev. A 13, 2123 (1976).

${ }^{2}$ W. Lamb and R. Retherford, Phys. Rev. 72, 241 (1947).

${ }^{3}$ A. Aspect, G. Roger, S. Reynaud, J. Dalibard, and C. CohenTannoudji, Phys. Rev. Lett. 45, 617 (1980).

${ }^{4}$ C. Cohen-Tannoudji, J. Dupont-Roc, and G. Grynberg, AtomPhoton Interactions: Basic Processes and Applications (WileyVCH, Weinheim, 2004).

${ }^{5}$ H. Giessen, J. D. Berger, G. Mohs, P. Meystre, and S. F. Yelin, Phys. Rev. A 53, 2816 (1996).

${ }^{6}$ C. Weisbuch, M. Nishioka, A. Ishikawa, and Y. Arakawa, Phys. Rev. Lett. 69, 3314 (1992).

${ }^{7}$ C. Weisbuch and C. Herman, Phys. Rev. B 15, 816 (1977).

${ }^{8}$ P. D. Ye, D. Weiss, R. R. Gerhardts, M. Seeger, K. von Klitzing, K. Eberl, and H. Nickel, Phys. Rev. Lett. 74, 3013 (1995).

${ }^{9}$ V. Kubrak, F. Rahman, B. Gallagher, P. Main, M. Henini, C. Marrows, and M. Howson, Appl. Phys. Lett. 74, 2507 (1999).

${ }^{10}$ M. Hara, A. Endo, S. Katsumoto, and Y. Iye, Phys. Rev. B 69, 153304 (2004).

${ }^{11}$ A. Nogaret, S. J. Bending, and M. Henini, Phys. Rev. Lett. 84, 2231 (2000).

${ }^{12}$ J. Reijniers and F. Peeters, J. Phys.: Condens. Matter 12, 9771 (2000).

${ }^{13}$ A. Matulis, F. Peeters, and P. Vasilopoulos, Phys. Rev. Lett. 72, 1518 (1994).

${ }^{14}$ A. Nogaret, D. N. Lawton, D. K. Maude, J. C. Portal, and M. Henini, Phys. Rev. B 67, 165317 (2003).

${ }^{15}$ J. A. K. Freire, A. Matulis, F. M. Peeters, V. N. Freire, and G. A. Farias, Phys. Rev. B 61, 2895 (2000).

${ }^{16}$ Magnetoelectric edge states are ignored here as they are nonradiative.

${ }^{17}$ The angle of orbits for which Lorentz and Zeeman oscillations have similar amplitudes is obtained by $\cos (\theta / 2)=l_{\text {Zeeman }} / l_{b}$. For the InAs structure described in the text $l_{\text {Zeeman }}=2.2 \mathrm{~nm}, l_{b}$ $=323 \mathrm{~nm}$, and $\theta=0.8^{\circ}$.

${ }^{18}$ D. K. K. Lee, J. T. Chalker, and D. Y. K. Ko, Phys. Rev. B 50, 5272 (1994).

${ }^{19}$ F. Evers, A. D. Mirlin, D. G. Polyakov, and P. Wölfle, Phys. Rev. B 60, 8951 (1999).

${ }^{20}$ I. Gradshteyn and I. Ryzhik, Tables of Integrals Series and Products (Academic, New York, 1965).

${ }^{21}$ A. Nogaret, Phys. Rev. Lett. 94, 147207 (2005).

${ }^{22}$ N. Bloembergen and R. Pound, Phys. Rev. 95, 8 (1954).

${ }^{23}$ P. Bösinger, E. Brun, and D. Meier, Phys. Rev. Lett. 38, 602 (1977).

${ }^{24}$ R. Dicke, Phys. Rev. 93, 99 (1954).

${ }^{25}$ C. L. Joseph, C. Calero, and E. M. Chudnovsky, Phys. Rev. B 70, 174416 (2004).

${ }^{26}$ E. M. Chudnovsky and D. A. Garanin, Phys. Rev. Lett. 89, 157201 (2002).

${ }^{27}$ C. L. Davis, V. K. Henner, A. V. Tchernatinsky, and I. V. Kaganov, Phys. Rev. B 72, 054406 (2005).

${ }^{28}$ A. Nogaret, N. J. Lambert, and F. M. Peeters, following paper, Phys. Rev. B 76, 075312 (2007).

${ }^{29}$ T. Jeltes, J. McNamara, W. Hogervorst, W. Vassen, V. Krachmalnicoff, M. Schellekens, A. Perrin, H. Ghang, D. Boiron, A. Aspect et al., Nature (London) 445, 402 (2007).

${ }^{30}$ P. Michler, A. Imamoglu, M. Mason, P. Carson, G. Strouse, and S. Buratto, Nature (London) 406, 968 (2000).

${ }^{31}$ E. Moreau, I. Robert, L. Manin, V. Thierry-Mieg, J. M. Gerard, and I. Abram, Phys. Rev. Lett. 87, 183601 (2001).

${ }^{32}$ W. Press, S. Teukolsky, W. Wetterling, and B. Flannery, Numerical Recipes in $C$ (Cambridge University Press, Cambridge, 1994). 